\title{
Improvement of Adhesion of Kevlar Fiber to Poly(methyl methacrylate)
}

\author{
Ming-Liang ChEn, Shigeyuki UeTA, and Motowo TAKAYANAGI \\ Department of Industrial Chemistry, Faculty of Engineering, \\ Kyushu Sangyo University, Matsukadai, \\ Fukuoka 813, Japan
}

(Received March 8, 1988)

\begin{abstract}
The surface of Kevlar fibers can be modified by some chemicals through metalation reaction in a solution of methylsulfinylcarbanion and dimethylsulfoxide (DMSO) [Takayanagi et al., Rep. Prog. Polym. Phys. Jpn., 28, 243 (1985)]. In this study, the functional groups introduced to the surface of Kevlar fibers were identified by means of ESCA, referring to the atomic ratios of unmodified Kevlar. The adhesion of the fibers to poly(methyl methacrylate) (PMMA) was evaluated by a single fiber pull-out test. The carboxymethylated and epoxy-reacted fibers showed the strongest affinity to PMMA compared with the untreated fiber and the one having a less-polar surface layer. After the pull-out test, the fiber was observed by SEM to survey the fracture mechanisms. It was observed by SEM that the fiber adhered to PMMA, resulting in both modes of fracture: one case was a strip of fiber skin torn out from the fiber surface and another case, the matrix resin of PMMA was fractured, the residue of which adhered to the fiber surface. Introduction of polar groups such as carboxy and hydroxy groups to the fiber surface layer was most effective in increasing pull out strength.
\end{abstract}

KEY WORDS Interfacial Adhesion / Poly(methyl methacrylate) / Kevlar /

Pull-Out Test /

Kevlar fiber has low density, high tensile strength and high Young's modulus. Nowadays it is widely used in fiber-reinforced composites. Studies on the adhesion of Kevlar fibers to polymer resins have been reported in various papers. ${ }^{1-3}$ Poly(methyl methacrylate) (PMMA) is light, strong in compression, but brittle in tensile deformation. Improved mechanical properties of PMMA should enhance application of its composites with Kevlar to tooth plates, for example. The properties of short fiber-reinforced composites are mainly determined by the properties of the matrix and fiber, the content of the fibers, fiber aspect ratio, fiber morphorogy and adhesive strength at the fiber-matrix interface. Among these factors, adhesion at fiber-matrix is one of the most important factors. In this study, the Kevlar fiber surface was treated by some chemicals through polymer metalation reaction in a solution of methylsulfinylcarbanion and dimethylsulfoxide and a single fiber pullout test was used to evaluate the adhesion of a fiber and PMMA.

\section{EXPERIMENTAL}

\section{Evaluation of Interfacial Adhesion}

The interfacial shear stress of fiber and matrix is calculated by the following formula:

$$
\tau_{\mathrm{i}}=\frac{\sigma R}{2 L}
$$

where $\sigma$ is tensile stress for the pull-out of a single fiber, $\tau_{\mathrm{i}}$ is the interfacial shear stress, $R$, the radius of the fiber and $L$, the immersion length of the fiber in matrix. When $\sigma_{\mathrm{f}}$ is the ultimate tensile strength of a fiber, the follow- 
ing formula is obtained from eq 1 :

$$
L_{\mathrm{c}}=\frac{\sigma_{\mathrm{f}} R}{2 \tau_{\mathrm{i}}}
$$

From eq 2, the critical immersion length of the fiber, $L_{\mathrm{c}}$, can be calculated. In the pull-out test, if the fiber immersion length $L$ is shorter than $L_{\mathrm{c}}$, the fiber is pulled out. Otherwise, the fiber is broken. After being pulled out, the fiber was studied by scanning electron microscope to survey the fracture mechanism.

\section{Treatment of Kevlar Fiber Surface by Chem-} icals

At first, the Kevlar fiber surface was washed with acetone. In a three neck flask being equipped with an $\mathrm{N}_{2}$ inlet, drying tube, stirrer, and a thermometer, $150 \mathrm{ml}$ of DMSO and $0.12 \mathrm{~g}$ of $\mathrm{NaH}$ were reacted at $30^{\circ} \mathrm{C}$ for $30 \mathrm{~min}$ and $70^{\circ} \mathrm{C}$ for $30 \mathrm{~min}$. The surface cleaned fiber was placed in the flask and metalation was conducted at $30^{\circ} \mathrm{C}$ for $10 \mathrm{~min}$. At this time, the surface of the fiber was partially metalationreacted and swollen by dimethyl sulfoxide (DMSO). The metalated fiber was rapidly soaked in a solution of reagent for introducing functional groups. The treated fiber was washed with acetone.

Epoxy reaction was conducted at $50^{\circ} \mathrm{C}$ for $1 \mathrm{~h}$ in the solution of $10 \mathrm{~g}$ of epoxy and $150 \mathrm{ml}$ of DMSO. Liquid epoxy resin, DER 383 of Dow Chemical Co. with 176-186 of epoxy equivalent was used.

Allylation was conducted at $30^{\circ} \mathrm{C}$ for $2 \mathrm{~h}$ in a solution of $25 \mathrm{~g}$ of allylbromide in $300 \mathrm{ml}$ of DMSO.

Ethoxycarbonylmethylation was conducted at $50^{\circ} \mathrm{C}$ for $50 \mathrm{~min}$ in a solution of $15 \mathrm{ml}$ of ethyl monobromoacetate in $200 \mathrm{ml}$ DMSO. The carboxymethylated fiber was obtained by deesterification of the ethoxycarbonylmethylated fiber with $1 N$ hydrochloric acid solution at room temperature for $1 \mathrm{~h}$.

These reactions are represented as follows ${ }^{4}$ :
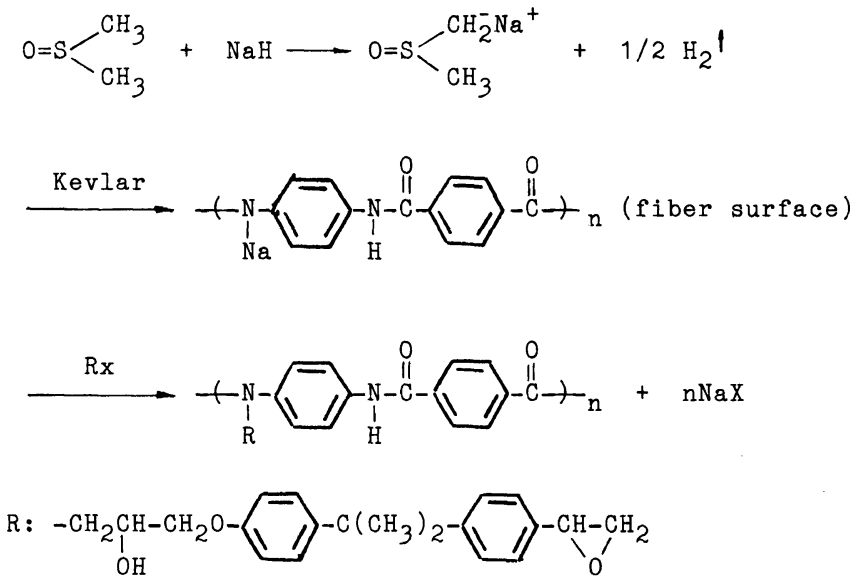

for epoxy reaction,

$-\mathrm{CH}_{2} \mathrm{CH}=\mathrm{CH}_{2}$ for allylation reaction,

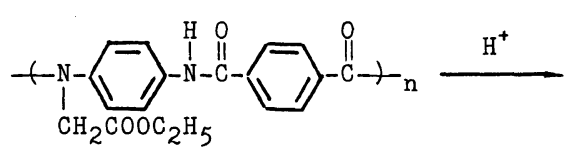

$-\mathrm{CH}_{2} \mathrm{COOC}_{2} \mathrm{H}_{5}$ for ethoxycarbonylmethylation, and for carboxymethylation:

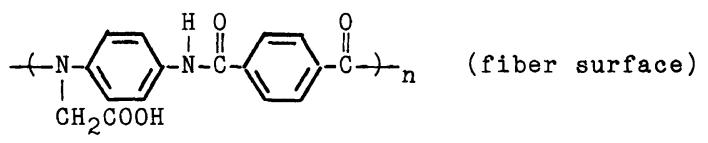


Preparation of a Single Fiber Pull-Out Samples

a) Preparation of PMMA-MMA. In a $200 \mathrm{ml}$ three neck flask equipped with an $\mathrm{N}_{2}$ inlet, stirrer, condenser with water circulation and a thermometer, $100 \mathrm{ml}$ of methyl methacrylate (MMA) and $0.6 \mathrm{~g}$ of benzoylperoxide (BPO) were reacted for $25 \mathrm{~min}$ at $70-75^{\circ} \mathrm{C}$, the content of PMMA being adjusted to about $25 \mathrm{wt} \%$. To terminate the polymerization, PMMA-MMA was placed in a refrigerator until use.

b) Preparation of a Single Fiber Pull-Out Samples. A single fiber was placed along the axis of a small glass tube, one end sealed by tape. PMMA-MMA was injected into the tube and the fiber immersion length in PMMA was controlled to $0.1-1 \mathrm{~mm}$. Then the tube was placed in a thermostat at $60^{\circ} \mathrm{C}$ for $8 \mathrm{~h}$ and at $85^{\circ} \mathrm{C}$ for $2 \mathrm{~h}$ for polymerization of remaining MMA. At room temperature, the single fiber pull-out test was conducted. The distance between the upper end of the fiber and horizontal upper surface of the PMMA resin was about $35 \mathrm{~mm}$. The pull-out rate was $5 \mathrm{~mm}$ $\min ^{-1}$ with a tensile tester, Tensilon. Figure 1 shows the preparation process of a single fiber

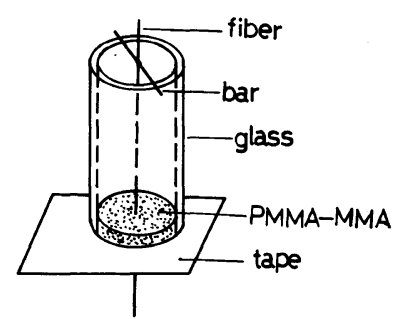

Figure 1. Preparation of a single fiber for a pull-out test.

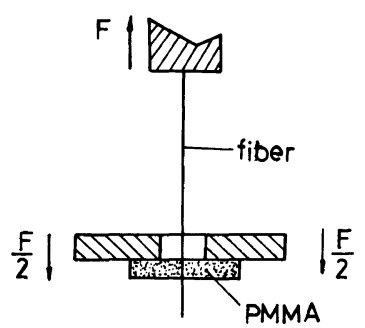

Figure 2. Layout of the pull-out test. pull-out test sample, and Figure 2, the layout of the pull-out test.

An optical microscope was used to measure the diameter of the fiber before the pull-out test and the thickness of PMMA resin, that is, the immersion length of the fiber after the test.

\section{RESULTS AND DISCUSSION}

Table I shows the ratios of the number of carbon atoms to that of nitrogen atoms $(\mathrm{C} / \mathrm{N})$ and oxygen atoms to nitrogen atoms $(\mathrm{O} / \mathrm{N})$ for various fibers calculated from ESCA spectra. During the treatment of the surface of the fibers, the number of nitrogen atoms did not change. The fact that $\mathrm{C} / \mathrm{N}$ and $\mathrm{O} / \mathrm{N}$ values of treated fibers were greater than those of the untreated fiber indicates that the surface of the fiber reacted with the chemical reagents. The number of epoxy groups introduced into the surface layer of fiber was the most. $\mathrm{C} / \mathrm{N}$ of carboxymethylated fiber was smaller than that of the ethoxycarbonylmethylated fiber, as the ethoxycarbonylmethyl groups were changed to carboxymethyl groups to some degree in the presence of protons.

Table I. Atom ratios of various fibers as calculated from ESCA

\begin{tabular}{lrr}
\hline Treatment of Kevlar & $\mathrm{C} / \mathrm{N}$ & $\mathrm{O} / \mathrm{N}$ \\
\hline Surface-cleaned & 13.60 & 5.57 \\
Allylated & 15.22 & 6.30 \\
Epoxy-reacted & 37.38 & 16.42 \\
Ethoxycarbonylmethylated & 17.96 & 6.31 \\
Carboxymethylated & 15.25 & 7.67 \\
\hline
\end{tabular}

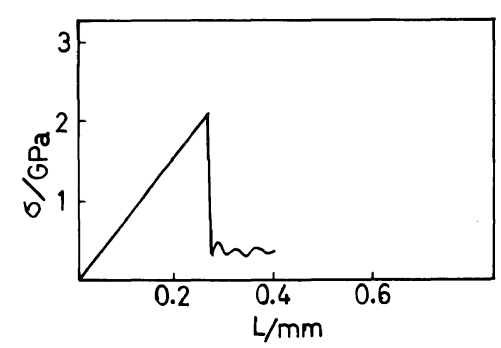

Figure 3. Typical curve of load-displacement of the pull-out test. 


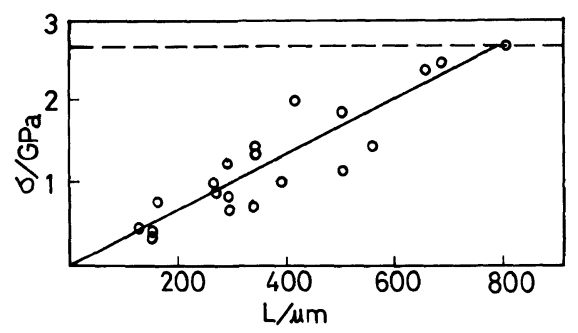

Figure 4. $\sigma$ vs. $L$ in the pull-out test for the cleaned Kevlar fiber.

Table II. Critical immersion length $\left(L_{\mathrm{c}}\right)$ and calculated interfacial shear stress $\left(\tau_{\mathrm{i}}\right)$ for different Kevlar/PMMA

\begin{tabular}{lcc}
\hline Treatment of Kevlar & $L_{\mathrm{c}} / \mu \mathrm{m}$ & $\tau_{\mathrm{i}} / \mathrm{MPa}$ \\
\hline Surface-cleaned & 780 & 11.25 \\
Metalation & 660 & 13.30 \\
Allylation & 620 & 14.15 \\
Epoxy-reacted & 480 & 18.28 \\
Ethoxycarbonylmethylated & 590 & 14.87 \\
Carboxymethylated & 480 & 18.28 \\
\hline
\end{tabular}

When fiber pull-out test was conducted, a load-displacement curve was recorded. Depending on the fibers whether being pulled out or broken, the load showed maximum tensile stress $\sigma_{\mathrm{m}}$ or tensile strength $\sigma_{\mathrm{f}}$. Figure 3 shows a typical load-displacement curve of the pull-out test. Figure 4 shows the relation of tensile stress $\sigma$ to immersion length $L$ for the cleaned Kevlar fiber. Table II summarizes the critical immersion length $L_{\mathrm{c}}$ and calculated mean interfacial shear stress $\tau_{\mathrm{i}}$ for the cleaned fiber and treated fibers. Adhesion of the fibers to PMMA can be evaluated by interfacial shear stress $\tau_{\mathrm{i}}$ and critical immersion length $L_{\mathrm{c}}$. The smaller the value of the immersion length $L_{\mathrm{c}}$ (the bigger the value of the interfacial shear stress $\tau_{\mathrm{i}}$ ), the greater the adhesion of the fiber to PMMA. For the cleaned fiber, $L_{\mathrm{c}}$ is $780 \mu \mathrm{m}$ and $\tau_{\mathrm{i}}$ is $11.25 \mathrm{MPa}$; but for the surface metalation fiber, $L_{\mathrm{c}}$ is $660 \mu \mathrm{m}$ and $\tau_{\mathrm{i}}$ is 13.3 $\mathrm{MPa}$. Thus, the adhesion of the surface of metalation fiber to PMMA is stronger than that of the cleaned fiber. As the surface of cleaned fiber is highly oriented and crystalline, (a)

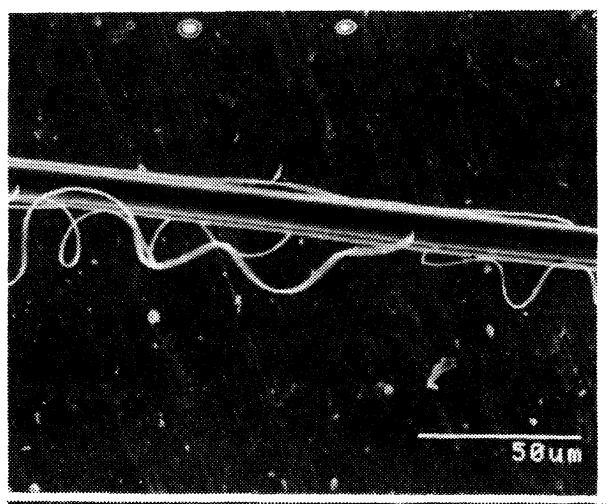

(b)

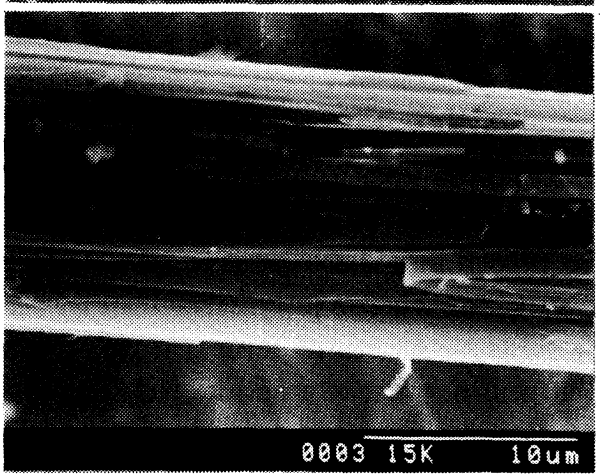

Figure 5. Scanning electron micrographs of untreated fibers pulled out from PMMA resin: (a) peeling off of the skin from a fiber; (b) remaining small amount of matrix resin on roughened surface of a fiber.

the metalation reaction acting to lower the high order of the fiber surface increases the affinity of the fiber to PMMA. In the case of the allylation fiber, $L_{\mathrm{c}}$ is $620 \mu \mathrm{m}$ and $\tau_{\mathrm{i}}$ is 14.15 $\mathrm{MPa}$. The allylation fiber is a little better than the metalation fiber in affinity to PMMA. Among these treatments of fibers, epoxyreaction and carboxymethylation are the most effective for improving the adhesion of the fiber to PMMA. This is thought due to the formation of hydrogen bonds between hydroxy groups in epoxy-reacted fiber or acid groups in carboxymethylated fiber and carbonyl groups in PMMA, in addition to the effect of the roughened interfacial layer during metalation reaction.

After being pulled out, the fibers were observed by scanning electron microscope. In 
general, when the fiber is pulled out, fracture occurs at the interface of the fiber and matrix or in matrix or at the surface of the fiber. In this study, observation of the fiber after being pulled out showed that the fracture mode was complex. Figure 5 shows typical SEM photographs of fibers after the pull-out test. Sometimes, the fracture of the fiber surface took place in the pull-out test. Figure 5(a) is such a case in which a thin fiber skin was stripped out of the fiber surface and formed a helical form. Figure 5(b) shows that the matrix was fractured so that some part of PMMA adhered to the surface of the fiber. As for the cleaned fiber, fracture often occurred on the surface of the fiber. When the fracture occurs in the matrix or at the surface of the fiber, the measured interfacial shear stress is lower than what it really is. Therefore, the above measured interfacial shear stress of various fibers and PMMA by the pull-out test may be lower in some cases than the actual values of in- terfacial adhesion. Even in the case of carboxymethylated and epoxy-reacted fibers, both fracture modes were observed. The difference in apparent adhesive strength as seen in Table II may be ascribed to the strength of interfacial layer; the crack generated in the layer grows either in the bulk of matrix resin or at the intersurface.

Acknowledgements. Permission to use ESCA by Professor T. Kajiyama, Kyushu University, is gratefully acknowledged.

\section{REFERENCES}

1. M. Takayanagi, S. Ueta and Y. Nishihara, Rep. Prog. Polym. Phys. Jpn., 28, 243 (1985).

2. D. Eagles, B. Blumentritt and S. Cooper, J. Appl. Polym. Sci., 20, 435 (1976).

3. M. Takayanagi and S. Ueta, Polym. Prepr. Jpn., 34, 1081 (1985).

4. M. Takayanagi and T. Katayose, J. Polym. Sci., Polym, Chem. Ed., 19, 1133 (1978). 